\title{
Hot snare vs. cold snare polypectomy for endoscopic removal of 4-10 mm colorectal polyps during colonoscopy: a systematic review and meta-analysis of randomized controlled studies
}

\section{다)(i) $(5)$}

\begin{abstract}
Authors
Institutions

1 Department of Gastroenterology and Hepatology, University of Kansas Medical Center, Kansas City, KS, USA

2 Department of Gastroenterology, Kansas City VA Medical Center, Kansas City, KS, USA

3 Division of Gastroenterology, University of Nevada, Las Vegas, NV, USA

4 Division of Gastroenterology, Humanitas Research Hospital \& Humanitas University, Milan, Italy

5 Nuovo Regina Margherita Hospital, Digestive Endoscopy Unit, Rome, Italy

6 Department of Medical and Surgical Sciences, S. OrsolaMalpighi Hospital, University of Bologna, Bologna, Italy
\end{abstract}

Ramprasad Jegadeesan ${ }^{1,2}$, Muhammad Aziz ${ }^{1,2}$, Madhav Desai ${ }^{1,2}$, Tharani Sundararajan², Venkata Subhash Gorrepati², Viveksandeep Thogulva Chandrasekar ${ }^{1,2}$, Mahendran Jayaraj ${ }^{3}$, Pratiksha Singh ${ }^{2}$, Ahmed Saeed ${ }^{2}$, Tarun Rai ${ }^{2}$, Abhishek Choudhary $^{2}$, Alessandro Repici ${ }^{4}$, Cesare Hassan ${ }^{5}$, Lorenzo Fuccio ${ }^{6}$, Prateek Sharma ${ }^{1,2}$

submitted 9.5.2018

accepted after revision 3.10 .2018

Bibliography

DOI https://doi.org/10.1055/a-0808-3680 |

Endoscopy International Open 2019; 07: E708-E716

(c) Georg Thieme Verlag KG Stuttgart · New York elSSN 2196-9736

Corresponding author

Prateek Sharma, MD, Department of Gastroenterology/

Hepatology and Motility, University of Kansas Medical

Center, Kansas City, KS, USA

Fax: +1-913-588-3877

psharma@kumc.edu

\section{ABSTRACT}

Introduction In recent years, cold snare polypectomy (CSP) has increasingly been used over hot snare polypectomy (HSP) for the removal of colorectal polyps $(4-10 \mathrm{~mm}$ in size). However, the optimal technique (CSP vs. HSP), in terms of complete polyp resection and complications, is uncertain. Our aim was to compare incomplete resection rate (IRR) of polyps and complications using CSP vs. HSP.

Methods Randomized controlled studies (RCTs) comparing CSP and HSP for removal of $4-10 \mathrm{~mm}$ colorectal polyps were considered. Studies were included in the analysis if they obtained biopsy specimens from the resection margin to confirm the absence of residual tissue and reported complications. IRR and complication rate were the outcome measures. Pooled rates were reported as Odds Ratios (OR) or risk difference with $95 \%$ Confidence Interval (Cl).

Results In total, three RCTs were included in the final analysis. A total of 1051 patients with 1485 polyps were randomized to either HSP group ( $n=741$ polyps) or CSP group ( $n=744$ polyps). The overall IRR did not differ between the two groups (HSP vs. CSP: $2.4 \%$ vs. $4.7 \%$; OR $0.51,95 \% \mathrm{Cl}$ $\left.0.13-1.99, P=0.33, I^{2}=73 \%\right)$. The HSP group had a lower rate of overall complications compared to the CSP group (3.7\% vs. 6.6\%; OR 0.53, $95 \% \mathrm{Cl} 0.3-0.94, P=0.03, P^{2}=$ $0 \%$ ). Polyp retrieval rates were not different between the two groups ( $99 \%$ vs. $98.1 \%$ ).

Conclusion Our results suggest that HSP and CSP techniques can be effectively used for the complete removal of 4-10 mm colorectal polyps; however, HSP has a lower incidence of overall complications.

\section{Introduction}

Colorectal cancer (CRC) is the third most common cancer in the US with 135-420 new cases per year [1]. Screening colonoscopy with polypectomy substantially reduces CRC incidence and mortality $[2,3]$. However, an unexpected high rate of incom- plete resection of colon polyps has been reported in some recent studies, and this has in turn been related to a higher risk of post-colonoscopy interval CRC [4].

Diminutive $(<5 \mathrm{~mm})$ and small $(6-9 \mathrm{~mm})$ polyps represent the vast majority of polyps removed at screening colonoscopy [5]. In theory, two major techniques are available for these le- 
sions, namely biopsy-forceps polypectomy and snare polypectomy. The former, however, has been associated with a much higher rate of incomplete resection, especially for small polyps, and its use is not generally recommended [6]. The latter may be further classified into hot (HSP) and cold (CSP) snare polypectomy. The basic difference is the use of a high frequency generator for HSP. Such use may, on the one hand, minimize immediate post-polypectomy bleeding by coagulation, but, on the other, it may also damage deeper vessels with increased risk of delayed bleeding $[7,8]$ or even perforation. For this reason, CSP is usually considered safer, while resulting in equivalent rates of complete resection, and its use has dramatically increased in recent years [9-12], due also to the development of specific CSP-snares. CSP- and HSP-incomplete resection rates (IRR) for $\leq 10 \mathrm{~mm}$ polyps range widely, being $0.5-6.4 \%$ and $1.2-7.4 \%$, respectively [12-15], and these estimates are much lower compared with forceps-polypectomy $[6,16]$. However, any advantage of one technique (HSP vs. CSP) over the other remains unclear [17].

The current European Society of Gastrointestinal Endoscopy (ESGE) guidelines recommend CSP as the preferred technique for removal of diminutive polyps (size $\leq 5 \mathrm{~mm}$ ) due to high rates of complete resection, adequate tissue sampling for histology, and low complication rates [18]. They also suggest CSP for sessile polyps $6-9 \mathrm{~mm}$ in size because of its superior safety profile, although evidence comparing efficacy with HSP is lacking [18].

Recently, randomized controlled studies (RCTs) have compared the efficacy of HSP with CSP for colorectal polyps ranging between 4 and $10 \mathrm{~mm}$ with a disparity in results [19-21]. Given this discrepancy and the lack of supporting evidence for current guidelines for polypectomy, we performed a systemic review and meta-analysis of available evidence with the objective to compare the IRR and adverse events between CSP and HSP when removing polyps between 4 and $10 \mathrm{~mm}$ in size.

\section{Materials and methods}

A comprehensive search was performed using PubMed, Embase, and abstracts of conferences presented at Digestive Diseases Week and the United European Gastroenterology Week. These sources were searched using the keywords "polypectomy", "cold polypectomy", "cold snare polypectomy", "hot snare polypectomy", "remnant adenoma", and "endoscopic mucosal resection" to extract studies up to December 2017. Only English language and human studies were searched. Related data suggested by PubMed were also searched. Two authors (RJ and MA) individually searched the databases for article screening. The abstracts were not blinded for authors, institutions or journals during review. Only data from randomized controlled trials comparing the efficacy of and adverse events related to the use of CSP with those of HSP (with or without submucosal injection) for removal of $4-10 \mathrm{~mm}$ colorectal polyps were included in our study. Such '4-10 mm' definition was preferred over the generally reported ' 6 - 9 mm' for small polyps, in order not to exclude relevant studies, and also include 4-, 5- or 10-mm polyps. In addition, we limited our inclusion to only those studies where biopsy specimens were obtain- ed from the post-polypectomy resection margin to confirm the absence of any residual polypoid tissue (i.e. endoscopic and histologic radical resection). If multiple articles were found from the same institution, then the most recent article was used for analysis. Letters to editors, case reports, case series, case-control, and cohort studies were excluded.

\section{Data collection and bias assessment}

Baseline demographic data (age, gender), number of study participants, number of polyps examined, morphology of polyps, histology of polyps, location of polyps, IRR, type of snare used, procedure time, and complication rates were extracted from each study. Paris classification [22] and Vienna classification [23] systems were used to assess the morphology and histology of polyps, respectively. Polyps were classified as either right-sided (if found in the cecum, ascending colon or transverse colon) or left-sided (if found in the descending colon, sigmoid coIon, or rectum). IRR was defined as the presence of any residual polypoid tissue in biopsied specimen post-polypectomy with either technique. Procedural time was defined as the time required from identification of polyp to complete resection of the polyp using either technique [19, 21].

Complications included immediate bleeding during the procedure, delayed bleeding after the procedure, and perforations of the intestinal wall. Immediate bleeding was defined as continuous hemorrhage usually for $\geq 30$ seconds immediately after polypectomy [19]. Delayed bleeding was defined as hemorrhage after colonoscopy requiring endoscopic hemostasis. All three studies have excluded patients who were treated with antithrombotic agents.

\section{Outcome measures}

The primary outcome of our study was IRR and secondary outcomes were complication rates, polyp retrieval rates, and procedure time.

\section{Risk of bias assessment}

The risk of bias was evaluated based on guidelines from the Cochrane Handbook for Systematic Reviews of Interventions [24] using the following: adequacy of random sequence generation, allocation concealment, blinding of the participant, blind outcome assessment, incomplete outcome data, and selective outcome reporting.

\section{Statistical analysis}

Pooled odds ratio (OR) and $95 \%$ confidence interval $(\mathrm{Cl})$ were calculated for primary and secondary outcomes. For pooled analysis of rare events, risk difference was used as an estimate to compare any possible detectable difference, when applicable. Heterogeneity of the study was assessed using the $R^{2}$ statistic [25]. Percentages of $25 \%\left(R^{2}=25\right), 50 \%\left(R^{2}=50\right)$, and $75 \%$ $\left(R^{2}=75\right)$ were considered to be a low, moderate, and high degree of heterogeneity, respectively. In the presence of substantial heterogeneity $\left(I^{2}>50 \%\right)$, a random effect model was used as the pooling method; otherwise, a fixed effect model was adopted as the pooling method. Statistical software used was 
Review Manager (RevMan) v.5.3 (The Cochrane Collaboration, Oxford, Oxfordshire, UK).

\section{Results}

A total of 244 studies were retrieved using the databases ( Fig. 1). After screening through the title, abstracts, and full texts, a total of three RCTs were selected [19-21]. All three studies were published between April 2017 and August 2017 ( $\vee$ Table 1). A total of 1051 patients were found to have 1485 small polyps (4-10 $\mathrm{mm}$ in size) which were randomized to either the HSP group ( $n=741$ polyps) or the CSP group ( $n=744$ polyps). Of 1485 colorectal polyps, 1266 polyps were included in the final analysis with 630 polyps in the HSP group and 636 polyps in the CSP group. Two RCTs $[19,21]$ included only neoplastic polyps for the final analysis while one RCT [20] included all colorectal polyps which were excised. The mean age of patients randomized to the CSP group was 64.1 years old, while those randomized to the HSP group was 65.3 years old, as described in two studies $[20,21]$. The mean percentages of males were $55.5 \%$ in the CSP group and $56.8 \%$ in the HSP group.

The risks of bias assessment are reported in $>$ Fig. 2 .

\section{Type of snare}

The snares used in these studies were: Captivator II $10 \mathrm{~mm}$ (Boston Scientific, Natick, MA, United States) [19,20], the Acusnare (Cook Medical, Bloomington, IN, USA) [20], SnareMaster $10 \mathrm{~mm}$ (Olympus Medical, Tokyo, Japan) [20, 21] and Exacto/9 mm (US Endoscopy, Mentor, OH, United States) [19]. For HSP, electro-cautery was performed with the ERBE ICC 200 electrosurgical generator (ERBE Electromedizin, Tübingen, Germany) $[20,21]$. Only one study mentioned the setting for cautery as fractionated cutting mode ENDO CUT Q (effect 3; cut duration 1; cut interval 3) [20]. Sub-mucosal injection before polypectomy was performed for both groups in one study [19], however, it was only performed for the HSP group in the other two studies $[20,21]$.

\section{Polyp characteristics}

Based on the morphology of polyps, 436 out of 691 (63.1\%) and 432 out of 685 (63.1\%) were classified as type 0 - I polyps, while 255 out of 691 (36.9\%) and 263 out 685 (38.4\%) were classified as type 0 - II in the CSP and HSP group, respectively. On a histological basis, the following polyps were removed: 1152 (78.1\%) tubular adenomas, 111 (7.5\%) advanced adenomas (prominent villous component and high grade dysplasia), $86(5.8 \%)$ sessile serrated polyps/adenoma, 83 (5.6\%) hyperplastic polyps and $43(2.9 \%)$ polyps without histological diagnosis. The locations of polyps were reported in all studies with a total of 757 out of 1485 (51\%) found to be right-sided. All three studies reported the average polyp sizes which were not significantly different on comparison. The size averaged between 5.4 and $8.3 \mathrm{~mm}$ as provided in $>$ Table 1 .

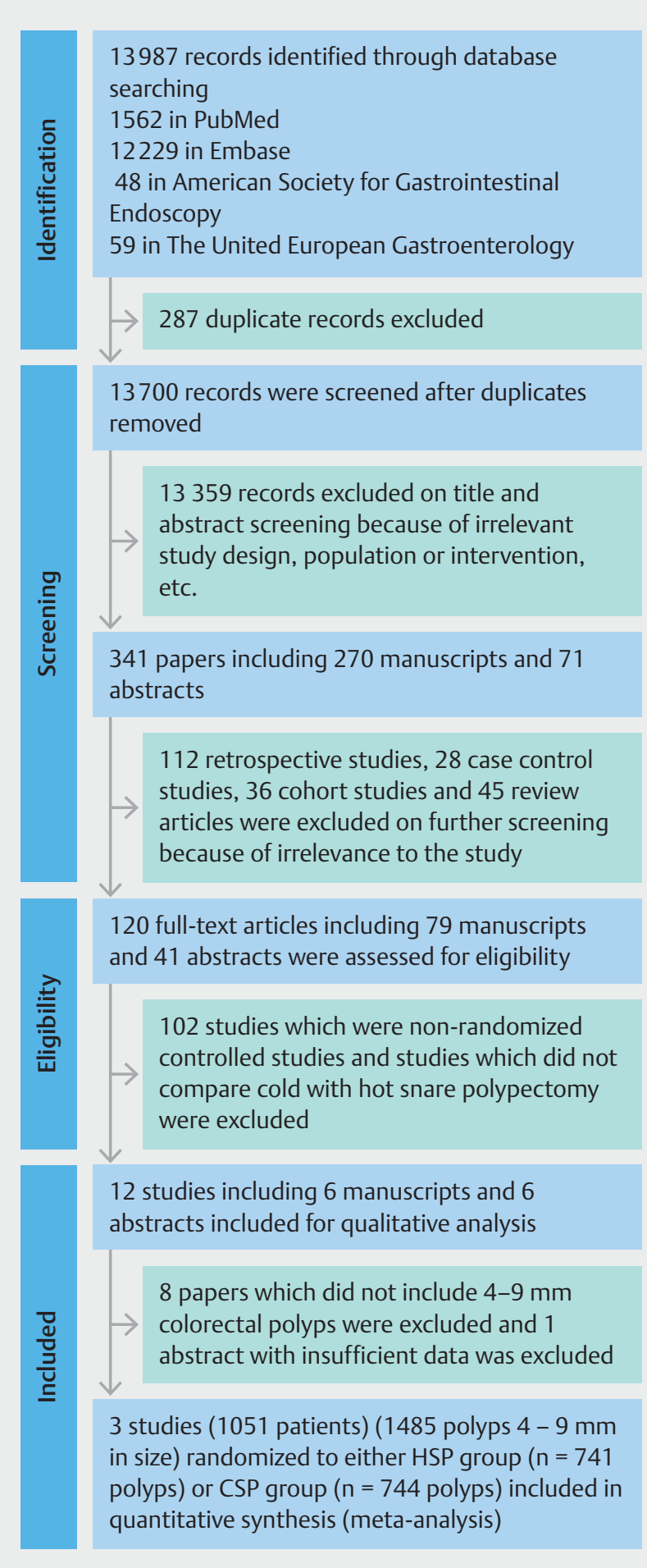

- Fig. 1 PRISMA flow diagram for selection of studies.

\section{Endoscopic mucosal resection}

Submucosal injection before polypectomy for endoscopic mucosal resection (EMR) was performed for both groups in one study [19]; however, it was only performed for selected polyps in the HSP group in the other two studies based on endoscopist preference $[20,21]$. The injected submucosal solution included 


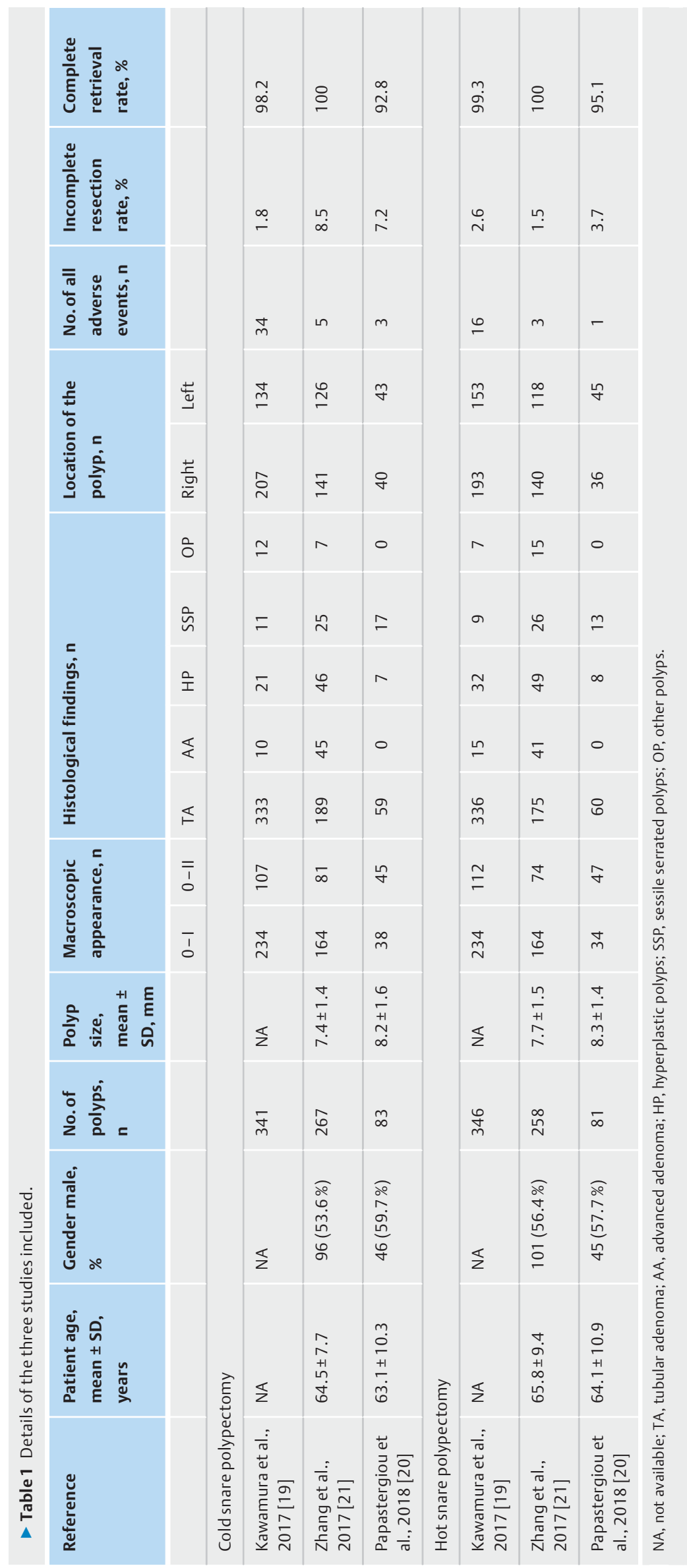




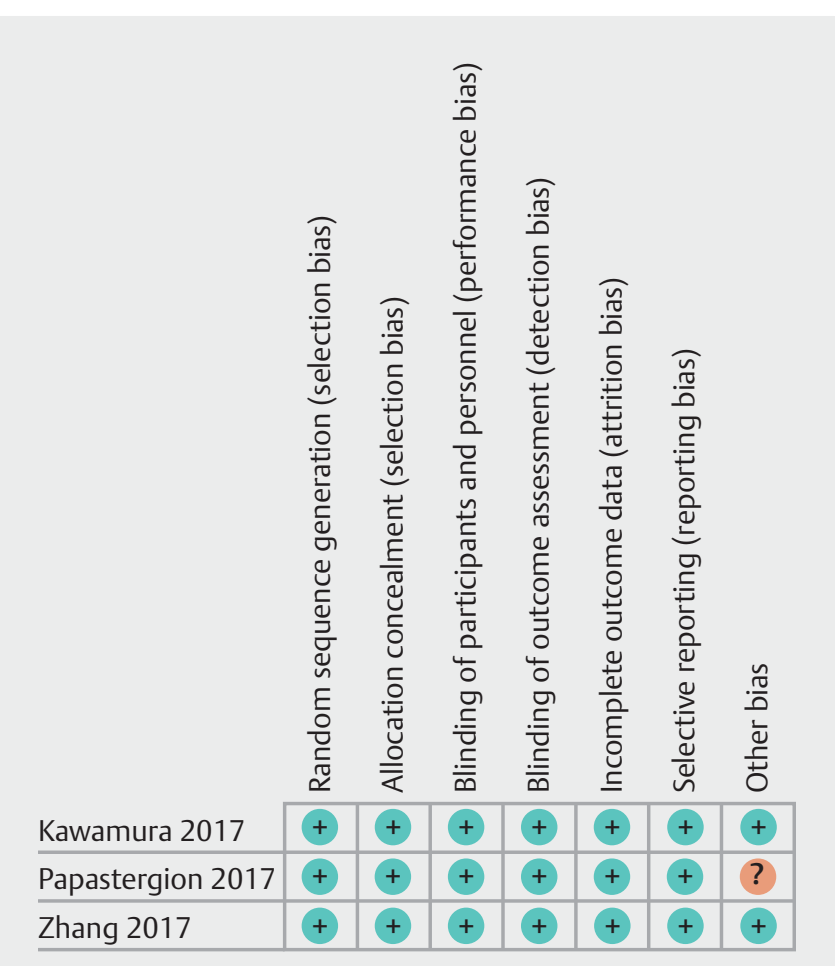

- Fig. 2 Risk of bias per Cochrane risk of bias assessment among studies included.

$1: 10000$ epinephrine in one of the studies that allowed submucosal injection for HSP only [21] while the other two studies did not use any epinephrine in the injected submucosal solution.

\section{Incomplete resection rate}

IRR was assessed via four-quadrant biopsy along with biopsy of the base of the post-polypectomy site in two studies [19,20] and biopsy of the left and right lateral margins in one study [21]. IRR was collectively found to be $2.4 \%(15 / 630)$ and $4.7 \%$ (30/636) for HSP and CSP group, respectively. The study heterogeneity was substantial $\left(I^{2}=73 \%\right)$, hence a random effects model was used to assess the difference in incomplete resection. The pooled odds ratio was 0.51 (95\% Cl 0.13-1.99, $P=$
0.33). The difference in IRR between HSP and CSP was not statistically significant ( $>$ Fig. 3 ).

\section{Adverse events}

All three studies reported adverse events (immediate procedural bleeding, delayed bleeding, and perforations). A total of 741 polyps were removed using HSP and 744 polyps were removed employing CSP. Clinically and statistically, a lower rate of overall adverse events was observed with HSP compared to CSP (20/ 546 [3.7\%] vs. 36/545 [6.6\%], pooled OR 0.53, 95\% Cl $0.3-$ $0.94, P=0.03 ; P^{2}=0 \%$ ) ( Fig. 4a).

When assessing adverse events separately, immediate bleeding rate was lower in the HSP group (18/546 [3.3\%]) compared to the CSP group (36/545 [6.6\%]), yielding a pooled OR 0.48 ( $95 \% \mathrm{Cl} 0.27-0.86, P=0.01 ; R^{2}=0 \%$ ) ( Fig. 4b). All patients with immediate post-polypectomy bleeding required endoscopic hemostasis to control bleeding and was clinically successful in all three studies. Two studies used hemostatic clip placement for endoscopic hemostasis $[20,21]$ while one study did not mention the technique used for endoscopic hemostasis [19].

The delayed bleeding rates were not different between the two groups (2/546 [0.4\%] vs. 0/545 [0\%]) ( Fig. 4c). Delayed bleeding occurred in only two patients who underwent HSP in one of the studies [19] while the other two studies did not have any occurrence of delayed bleeding in either group. Since delayed bleeding was considered a "rare event" among the studies included, we used pooled risk difference as pooled estimate to detect if any difference existed between the groups. Pooled risk difference was zero and results were not statistically significant $(P=0.45)$.

No case of perforation was reported in either group in any of the three studies. Preventive hemostasis, defined as prophylactic coagulation of vessels or red spots in the ulcer or clipping of a non-bleeding post-polypectomy mucosal defect, was allowed in the HSP group, but not in the CSP group in one study [19].

\section{Polyp retrieval rate}

The polyp retrieval rate was not clinically or statistically significantly different between the two groups. Collective retrieval rates were $99 \%$ for the HSP group and $98.1 \%$ for the CSP group

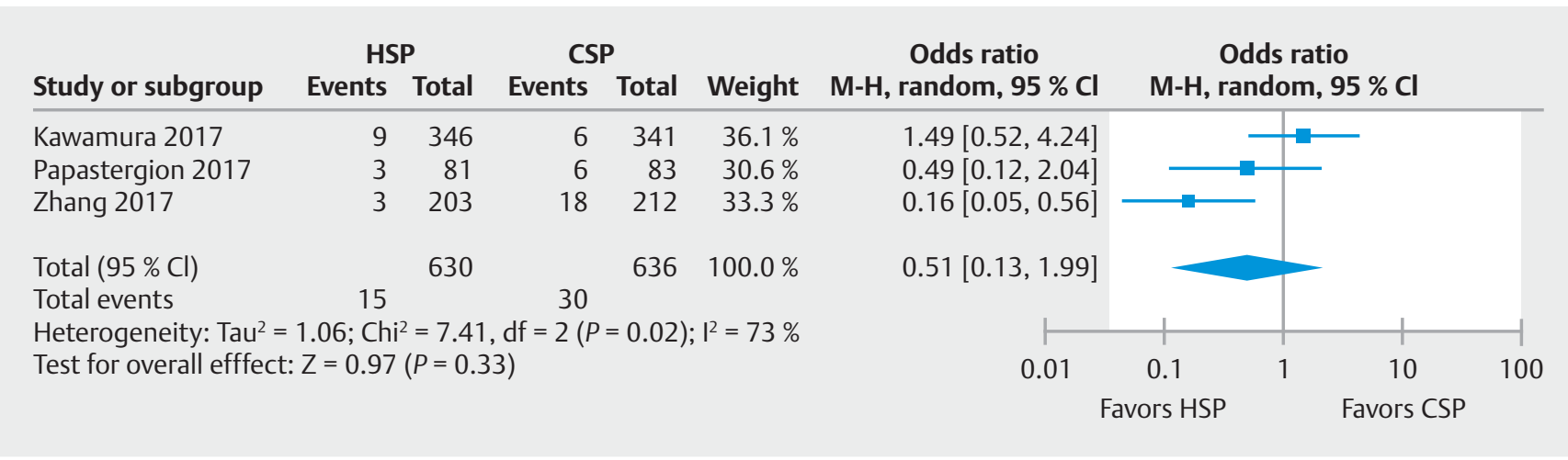

- Fig. 3 Forest plot of all RCTs assessing incomplete resection rate (IRR) between HSP/EMR and CSP group. 


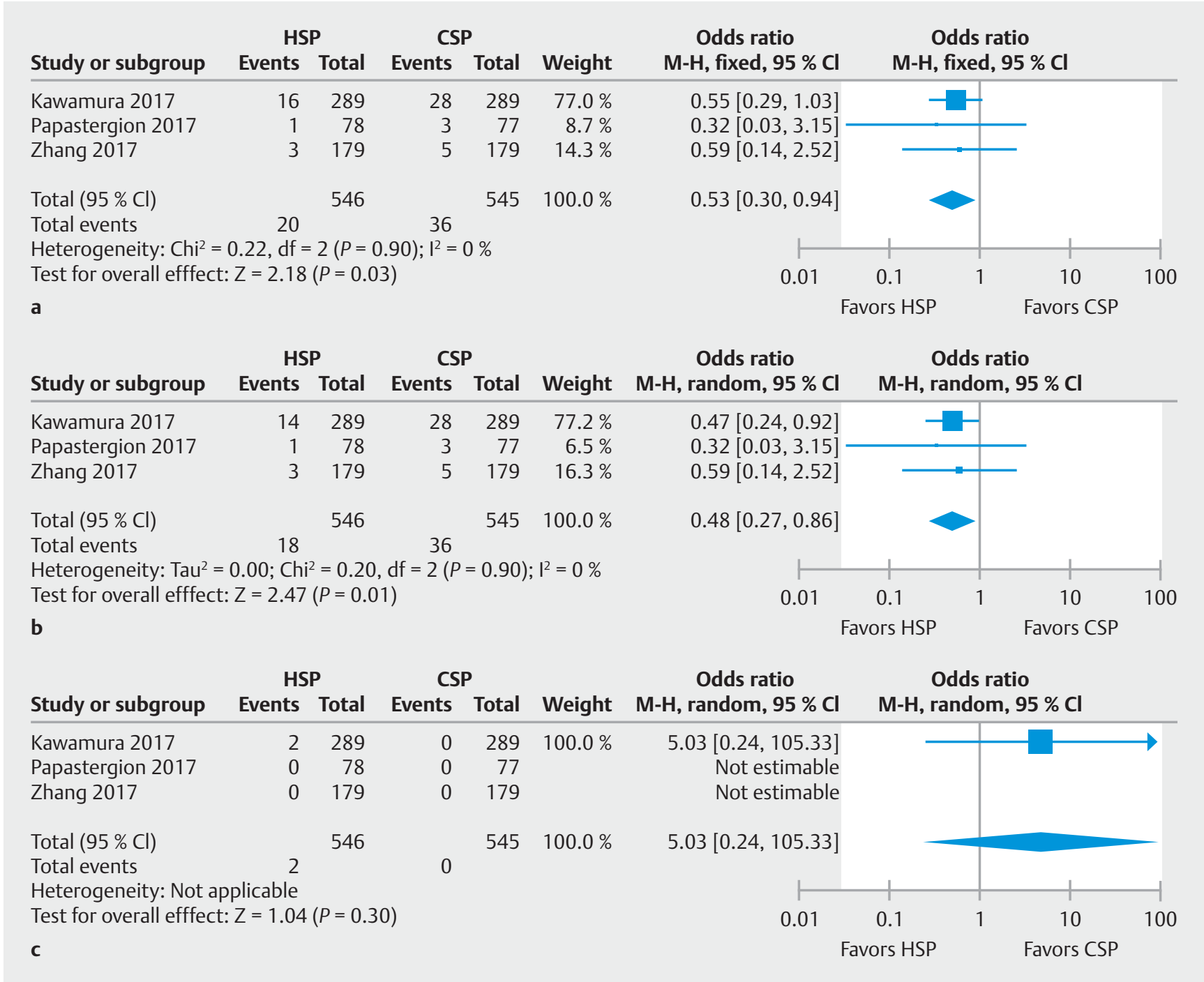

- Fig. 4 Forest plot of all RCTs assessing: $\mathbf{a}$ total complication rate; $\mathbf{b}$ immediate bleeding rate; $\mathbf{c}$ delayed bleeding rate between HSP/EMR and CSP group.

yielding a pooled OR of $1.9\left(95 \% \mathrm{Cl} 0.74-4.83, P=0.18 ; I^{2}=0 \%\right)$ as shown in $\mathbf{F i g} \mathbf{5}$.

\section{Procedure time}

Procedural time was defined as the time required from identification of polyp to complete resection of the polyp using either technique $[19,21]$. Only two studies reported procedural time for polypectomies. Kawamura et al. reported median times of 83 seconds and 60 seconds for the HSP and CSP group, respectively [19]. Zhang et al. had comparatively longer times for their polypectomies with mean procedural times of $330 \mathrm{sec}$ onds and 282 seconds, respectively for the HSP and CSP group [21].

\section{Discussion}

The results of this meta-analysis suggest that there is no significant difference between cold and hot snare in achieving a complete resection of $4-10 \mathrm{~mm}$ colorectal polyps, but there is a higher rate of adverse events with cold snare, albeit this is limited to immediate post-polypectomy bleeding that was amenable to endoscopic treatment in all of the cases.

The main result of our analysis is the equivalence between the two techniques in achieving a complete endoscopic resection. This is clinically relevant as completeness of resection represents by far the dominant end point when approaching the risk/benefit ratio of endoscopic resection for sub-centimetric lesions. Our meta-analysis, however, showed that there is residual uncertainty on the equivalence between the two techniques, as shown by the very high heterogeneity level in this estimate. In detail, in two of the three studies, the rate of incomplete resection was 2 - to 6 -fold higher with cold snare than hot 


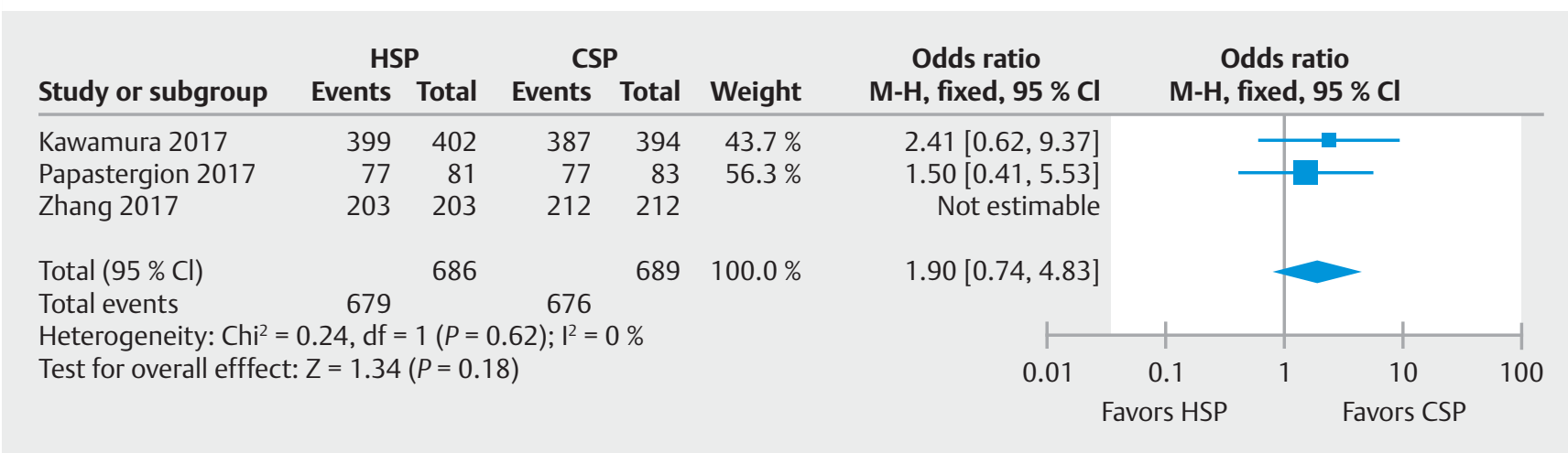

- Fig. 5 Forest plot of all RCTs assessing polyp retrieval rate between HSP/EMR and CSP group.

snare $[20,21]$, while in the remaining study a very low rate of incomplete resection was shown in both of the arms [19]. The risk of incomplete resection with cold snare, was however $<10 \%$ in all three trials, marginalizing the clinical impact of a possible superiority of the hot snare technique for such an end point. For instance, the overall $4.7 \%$ rate of incomplete resection with cold-snaring is much lower than the nearly $20 \%$ estimated for forceps biopsy when removing diminutive polyps [16, 26].

Adverse events related to both CSP and HSP include risk of bleeding (immediate bleeding during the procedure or delayed bleeding after colonoscopy) and intestinal perforation. In a large, prospective, non-randomized study, Repici et al. reported the rate of immediate bleeding for CSP to be $1.8 \%[10,27]$. The rate for immediate bleeding has been reported to be 0 $1.4 \%$ for HSP $[13,15]$. The immediate bleeding rates in our meta-analysis were $6.6 \%$ and $3.3 \%$ for CSP and HSP groups, respectively. There were two major limitations in the included studies which could have affected immediate bleeding rate between the two techniques. One of the three studies included allowed submucosal injection which also included epinephrine in the HSP group only [21], and which could have decreased immediate post-polypectomy bleeding in the HSP group. Also, only one study [19] defined immediate bleeding to be continuous bleeding for $\geq 30$ seconds while the other two studies did not provide any specific definition. Immediate oozing is destined to occur after cold snare polypectomy due to lack of coagulation current. In most cases, it is a slow, capillary bleeding that spontaneously stops and does not need intervention [13, 15].

The incidences of delayed post-polypectomy bleeding observed in previous prospective studies were $0.6-2 \%$ and $0 \%$ for the HSP and CSP groups, respectively $[10,15,28,29]$. The delayed bleeding rates in our study were $0.3 \%$ and $0 \%$ for the HSP and CSP groups, respectively, which are consistent with previous studies. As this was a rare event in our included studies, we used risk difference as pooled estimate; pooled risk difference was zero and results were not statistically significant $(P=0.45)$ suggesting there was no difference between either method. Colonic perforation has been reported to be below $1 \%$ using HSP and $0 \%$ with CSP $[30,31]$. The overall adverse events for our meta-analysis were lower for the HSP group compared to CSP, with sub-analysis revealing immediate postprocedural bleeding to be lower for the HSP group. Clinically, this does not have any major significance as immediate bleeding is usually self-limiting and managed with observation on the majority of occasions or with post-procedural clipping immediately after polyp removal $[10,32]$. Since all three studies excluded patients treated with antithrombotic agents, the use of antithrombotic agents did not have any influence on post-polypectomy bleeding.

Several factors pose limitations to our meta-analysis. Firstly, despite a wide literature search on the main electronic databases, only three RCTs were available in the literature which were evaluated and compared in this systematic review and meta-analysis. Secondly, there was no follow-up colonoscopy for these patients to confirm "true" complete resection as polyps may recur in previously biopsied negative margin sites. Further studies are needed that not only biopsy the resection margins to confirm complete removal but also assess the patients at a followup surveillance colonoscopy and biopsy the scar area to determine the IRR. The post-polypectomy sites can also be evaluated by performing endoscopic mucosal resection of the $1-3 \mathrm{~mm}$ margin around the resection site to determine IRR as suggested by Matsuura et al. [33]. Third, the rates of incidence of colon cancer and mortality from colon cancer were not assessed in these studies which undermines the true efficacy of removing small colorectal polyps. Large, multicenter long-term studies are needed to assess the incidence of colon cancer when comparing the two techniques in removing small colorectal polyps. Moreover, two studies reported four-quadrant biopsy along with biopsy of base of polypectomy site compared to one study that biopsied only left and right resected margins to display any residual polypoid tissue. This can imply potential bias and possibly false IRR. There is potential bias involved with different physicians as each endoscopist has a different skill set with different procedural times. There was also substantial heterogeneity noted between the three included studies which could be partially explained by different types of snare used between studies for polypectomy and the difference in the utility of submucosal injection between CSP and HSP groups in the included studies. Also, immediate bleeding was only defined in one study, while the other studies used the term "intra-procedural 
bleeding" that was summed together as "immediate bleeding" for our systematic review. Finally, we only searched articles published in the English language and may have missed relevant studies published in other languages.

Despite the above limitations, the main strength of this meta-analysis is the fact that all three studies being compared were randomized controlled trials (RCTs) with a low risk of bias based on the Cochrane risk of bias assessment scale that demonstrated incomplete resection rate (IRR) using post-polypectomy biopsy to confirm residual tissue. Furthermore, all RCTs assessed polyp size between 4 and $10 \mathrm{~mm}$ which is in accordance with our study objective and further decreased the bias among polyp sizes.

In conclusion, our study findings show that there is no statistically significant difference between the two techniques, although the rate of overall complications is higher with CSP; however, this is self-limiting. Based on currently available RCTs, we suggest that, clinically, either CSP or HSP can be safely used as one of the standard techniques for the resection of 4 $10 \mathrm{~mm}$ colorectal polyps. More studies are needed in future to assess recurrence of polyps and incidence of cancer to further gauge the efficacy of these two techniques.

\section{Competing interests}

None

\section{References}

[1] American Cancer Society. Cancer Facts \& Figures 2017. Available from: https://www.cancer.org/content/dam/cancer-org/research/ cancer-facts-and-statistics/annual-cancer-facts-and-figures/2017/ cancer-facts-and-figures-2017.pdf [cited 21 December 2017]

[2] Winawer S, Zauber A, Ho M et al. Prevention of colorectal cancer by colonoscopic polypectomy. NEJM 1993; 329: 1977-1981

[3] Zauber A, Winawer S, O'Brien M et al. Colonoscopic polypectomy and long-term prevention of colorectal-cancer deaths. NEJM 2012; 366: 687-696

[4] Pohl H, Srivastava A, Bensen S et al. Incomplete polyp resection during colonoscopy - results of the Complete Adenoma Resection (CARE) study. Gastroenterology 2013; 144: 74-80.e1

[5] Hassan C, Pickhardt P, Kim D et al. Systematic review: distribution of advanced neoplasia according to polyp size at screening colonoscopy. Aliment Pharmacol Ther 2010; 31: 210-217

[6] Gómez V, Badillo R, Crook J et al. Diminutive colorectal polyp resection comparing hot and cold snare and cold biopsy forceps polypectomy. Results of a pilot randomized, single-center study (with videos). Endosc Int Open 2014; 03: E76-E80

[7] Metz A, Moss A, Mcleod D et al. A blinded comparison of the safety and efficacy of hot biopsy forceps electrocauterization and conventional snare polypectomy for diminutive colonic polypectomy in a porcine model. Gastrointest Endosc 2013; 77: 484-490

[8] Yamashina T, Fukuhara M, Maruo T et al. Cold snare polypectomy reduced delayed postpolypectomy bleeding compared with conventional hot polypectomy: a propensity score-matching analysis. Endosc Int Open 2017; 05: E587-E594
[9] Piraka C, Saeed A, Waljee A et al. Cold snare polypectomy for nonpedunculated colon polyps greater than $1 \mathrm{~cm}$. Endosc Int Open 2017; 05: E184-E189

[10] Repici A, Hassan C, Vitetta E et al. Safety of cold polypectomy for $<10 \mathrm{~mm}$ polyps at colonoscopy: a prospective multicenter study. Endoscopy 2011; 44: 27-31

[11] Chukmaitov A, Bradley C, Dahman B et al. Polypectomy techniques, endoscopist characteristics, and serious gastrointestinal adverse events. J Surg Oncol 2014; 110: 207-213

[12] Aslan F, Camci M, Alper E et al. Cold snare polypectomy versus hot snare polypectomy in endoscopic treatment of small polyps. Turk J Gastroenterol 2014; 25: 279-283

[13] Ichise $Y$, Horiuchi A, Nakayama Y et al. Prospective randomized comparison of cold snare polypectomy and conventional polypectomy for small colorectal polyps. Digestion 2011; 84: $78-81$

[14] Horiuchi A, Nakayama Y. 587g: Prospective randomized comparison of cold snare polypectomy and conventional polypectomy. Gastrointest Endosc 2010; 71: AB127

[15] Paspatis G, Tribonias G, Konstantinidis K et al. A prospective randomized comparison of cold vs hot snare polypectomy in the occurrence of postpolypectomy bleeding in small colonic polyps. Colorectal Dis 2011; 13: e345-e348

[16] Efthymiou M, Taylor A, Desmond P et al. Biopsy forceps is inadequate for the resection of diminutive polyps. Endoscopy 2011; 43: 312-316

[17] Fujiya $M$, Sato $H$, Ueno $N$ et al. Efficacy and adverse events of cold vs hot polypectomy: A meta-analysis. World J Gastroenterol 2016; 22: 5436

[18] European Society of Gastrointestinal Endoscopy (ESGE). Colorectal polypectomy and endoscopic mucosal resection (EMR). 2018: Available from: http://www.esge.com/colorectal-polypectomy-and-endoscopic-mucosal-resection-emr-esge.html [cited 5 January 2018]

[19] Kawamura T, Takeuchi $Y$, Asai S et al. A comparison of the resection rate for cold and hot snare polypectomy for 4-9 mm colorectal polyps: a multicentre randomised controlled trial (CRESCENT study). Gut 2018; 67: 1950 - 1957

[20] Papastergiou V, Paraskeva K, Fragaki M et al. Cold versus hot endoscopic mucosal resection for nonpedunculated colorectal polyps sized 6-10 mm: a randomized trial. Endoscopy 2018; 50: 403-411

[21] Zhang Q, Gao P, Han B et al. Polypectomy for complete endoscopic resection of small colorectal polyps. Gastrointest Endosc 2018; 87: $733-740$

[22] Endoscopic Classification Review Group. Update on the Paris Classification of superficial neoplastic lesions in the digestive tract. Endoscopy 2005; 37: $570-578$

[23] Schlemper R. The Vienna classification of gastrointestinal epithelial neoplasia. Gut 2000; 47: 251-255

[24] Shuster J. Review: Cochrane Handbook for Systematic Reviews for Interventions, Version 5.1.0, published 3/2011. Julian P.T. Higgins and Sally Green, Editors. Res Synth Methods 2011; 2: $126-130$

[25] Higgins J, Thompson SG, Deeks J] et al. Measuring inconsistency in meta-analyses. BMJ 2003; 327: $557-560$

[26] Liu S, Ho S, Krinsky M. Quality of polyp resection during colonoscopy: are we achieving polyp clearance? Dig Dis Sci 2012; 57: 1786-1791

[27] Levin T, Zhao W, Conell C et al. Complications of colonoscopy in an integrated health care delivery system. Ann Intern Med 2006; 145: 880

[28] Sorbi D, Norton I, Conio M et al. Postpolypectomy lower GI bleeding: Descriptive analysis. Gastrointest Endosc 2000; 51: 690-696

[29] Park S, Lee M, Jeong S et al. Prospective analysis of minor adverse events after colon polypectomy. Dig Dis Sci 2017; 62: 2113-2119 
[30] Luigiano C, Consolo P, Scaffidi M et al. Endoscopic mucosal resection for large and giant sessile and flat colorectal polyps: a single-center experience with long-term follow-up. Endoscopy 2009; 41: 829-835

[31] Doniec M, Löhnert M, Schniewind B et al. Endoscopic removal of large colorectal polyps. Dis Colon Rectum 2003; 46: 340 - 348

[32] Heldwein W, Dollhopf M, Rösch T et al. The Munich Polypectomy Study (MUPS): Prospective analysis of complications and risk factors in 4000 colonic snare polypectomies. Endoscopy 2005; $37: 1116$ 1122

[33] Matsuura N, Takeuchi Y, Yamashina T et al. Incomplete resection rate of cold snare polypectomy: a prospective single-arm observational study. Endoscopy 2017; 49: $251-257$ 